\title{
End-effector pose estimation of the Monash Epicyclic-Parallel Manipulator through the visual observation of its legs
}

\author{
Alessia Vignolo ${ }^{1}$, Sébastien Briot $^{1}$, Philippe Martinet ${ }^{1,2}$, Chao Chen ${ }^{3}$
}

\begin{abstract}
Past research works have shown that it was possible to evaluate the end-effector pose of parallel robots by vision. First of all, it was proposed to directly observed the end-effector. However, this observation may be not possible (e.g. in the case of a haptic device for which the end-effector may be hidden by the user hand). Therefore, it has been proposed another type of end-effector pose estimation based on the observation of the directions of the legs. Even interesting results were obtained, this method is not suitable for some particular parallel robot families (e.g. the Monash Epicyclic-Parallel Manipulator, MEPaM). This paper proposes a new approach for the estimation of the endeffector pose: by observing the mechanism legs, it is possible to extract the Plücker coordinates of their lines and determine the end-effector pose. The new end-effector pose estimation method is applied to the MEPaM. All results are validated on a MEPaM simulator created using ADAMS/Controls and interfaced with Matlab/Simulink.
\end{abstract}

\section{INTRODUCTION}

If compared to serial robots, parallel robots have several advantages : higher speeds, higher dynamic performances and larger payload-to-weight ratio [1]. One of the disadvantages is that their control is tricky because of the high kinematic coupling, clearance effects in joints and manufacturing errors which deteriorate stability and accuracy.

In order to reduce this stability and accuracy degradation, which is due to the complex kinematic structure of the robot, sensor-based control may be used.

Vision-based control has shown its ability for estimating and controlling the end-effector pose, e.g. by the direct observation of the end-effector pose [2], [3], [4]. However, in some cases the direct observation of the end-effector may be impossible or difficult (for instance, with a haptic device where the user could prevent the observability of the endeffector). A good alternative for parallel robots is to observe the robot legs. In this case, by a direct measure of an internal property acquired through an external sensor, i.e. the robot leg configuration observed by vision, this technique allows to estimate the end-effector pose [5] which is an external property.

This method has been applied in [6] for the control of a Gough-Stewart (GS) platform [7]: the visual primitives were

\footnotetext{
This work was supported by the French Federation AtlanSTIC

${ }^{1}$ A. Vignolo, S. Briot, and P. Martinet are with the Institut de Recherche en Communications et Cybernétique de Nantes (IRCCyN), UMR CNRS 6597, Nantes, France

${ }^{2} \mathrm{P}$. Martinet is also with the Ecole Centrale de Nantes, Nantes, France

${ }^{3} \mathrm{C}$. Chen is with Monash University, Clayton, Australia

represented by the leg directions and the end-effector pose estimation was obtained from their reconstruction from the image. The same method was then applied to the Adept Quattro and other robots of the same family [8], [9].

However, as proven in [6], the approach cannot be applied to any type of robot family: for instance it was not possible to estimate the pose of a particular family of parallel robots for which the first joints of the legs are prismatic joints whose directions are all parallel [9] that. For example, in the case of

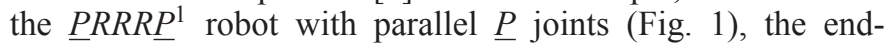
effector pose can not be reconstructed by the observation of leg directions $\underline{\mathbf{u}}_{i}^{2}$ only as, for the same values of $\underline{\mathbf{u}}_{1}$ and $\underline{\mathbf{u}}_{2}$, infinite possible end-effector poses can be found. In order to overcome this problem, a solution would be to use more information contained in the image, such as the Plücker coordinates of the lines passing through the legs. It will be shown in this paper that by observing the leg projection in the camera frame, it is possible to extract not only the leg direction but also the Plücker coordinates of the leg. Using the Plücker coordinates of the lines passing through the legs for the visual servoing is equivalent to use the leg direction plus their distance and position with respect to the camera frame. Thus, the line passing through the legs are fully defined. Estimating the end-effector pose in the case of the $\underline{P R R R} \underline{P}$ robot of Fig. 1 is similar to finding the intersection point of the lines $\mathcal{L}_{1}$ and $\mathcal{L}_{2}$ passing through the legs. In this paper the new end-effector pose estimation approach based on the use of the Plücker coordinates of the lines passing through the legs is introduced and it is applied to the MEPaM [10], a haptic device based on a six-degrees-of-freedom (dof) parallel kinematic machine.

\section{MEPAM}

\section{A. Architecture description}

MEPaM has an innovative design (Fig. 2). The actuators are mounted on the base instead of the moving limbs, reducing their mass and inertia. It has three legs and can preserve six-dof thanks to planetary-belt systems, which transmit power to the moving legs. There are three planetary-belt systems, and each one provides two-dof in a plane and is driven by two motors: a lower motor drives the lever arm A via a short stiff belt, and a upper motor drives the sun pulley via a long stiff belt. The planetary-belt system ends with the lever arm B, whose end is

\footnotetext{
${ }^{1}$ In the following of the paper, $R$ and $P$ stand for passive revolute and prismatic joints, respectively, while $\underline{R}$ and $\underline{P}$ stand for active revolute and prismatic joints, respectively.

${ }^{2}$ In the paper, $\underline{\mathbf{u}}_{i}$ stands for unit vector.
} 


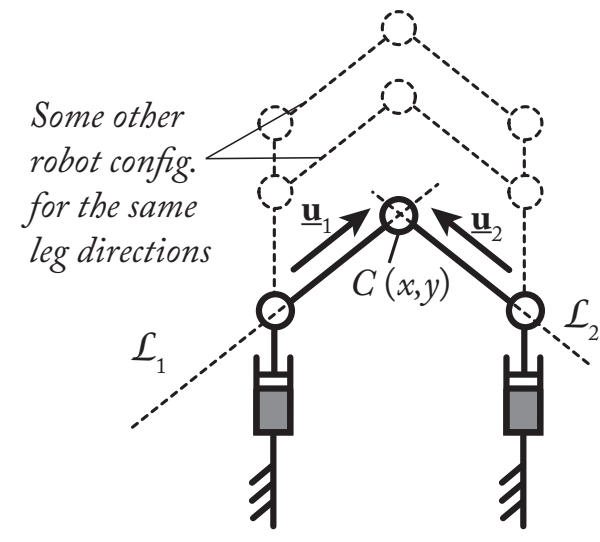

Fig. 1. The PRRRP robot

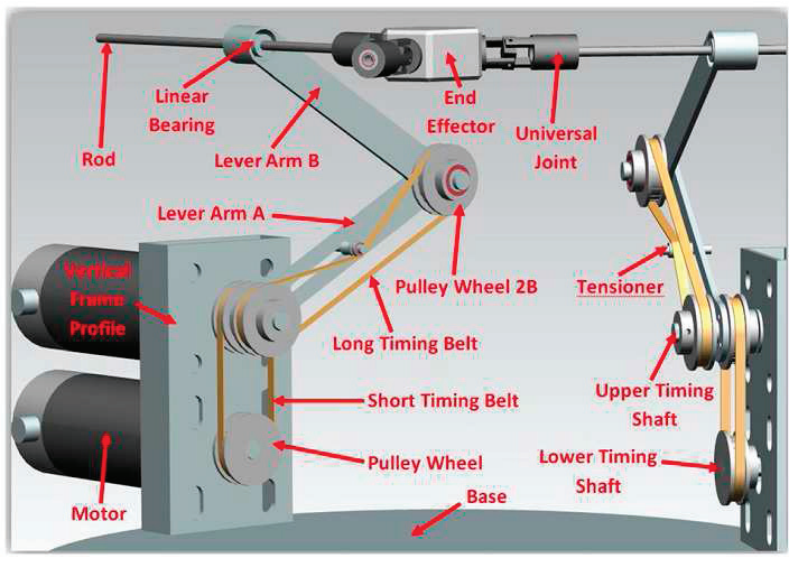

Fig. 2. Virtual model of MEPaM (one leg is hidden for clarity)

attached to a cylindrical joint perpendicular to the driving plane of the system. Then, one universal joint links every cylindrical joint to a vertex of the triangular end-effector.

\section{B. Forward kinematics of MEPaM based on the actuator angles and singularities}

The forward kinematics model of MEPaM based on the actuator angles and its singularities have been already analyzed in [10]. The serial and parallel singularities can be found from the analysis of the rank deficiency of the serial and parallel kinematic Jacobian matrices, defined through the relation

$$
\mathbf{J}_{S} \dot{\Theta}=\mathbf{J}_{P} \mathbf{t}
$$

where $\mathbf{J}_{S}$ and $\mathbf{J}_{P}$ are the serial and parallel kinematic Jacobian matrices, respectively, $\dot{\Theta}$ is the vector of active joints rates and $\mathbf{t}$ is the twist of the moving platform. Matrix $\mathbf{J}_{S}$ is rank deficient when there is serial singularity: in such a case for the MEPaM, the arms are fully extended or folded, and, in such a configuration, the triangular end-effector is not able to move in the arms direction, and it loses one-dof.

Concerning the parallel singularities which appear when the matrix $\mathbf{J}_{P}$ is rank deficient, in [10] they were determined from geometric conditions derived by Grassmann-Cayley algebra (GCA) [11]. The procedure, omitted for reason of brevity, leads to some geometric conditions for MEPaM's singularities, which are shown in Fig. 3.
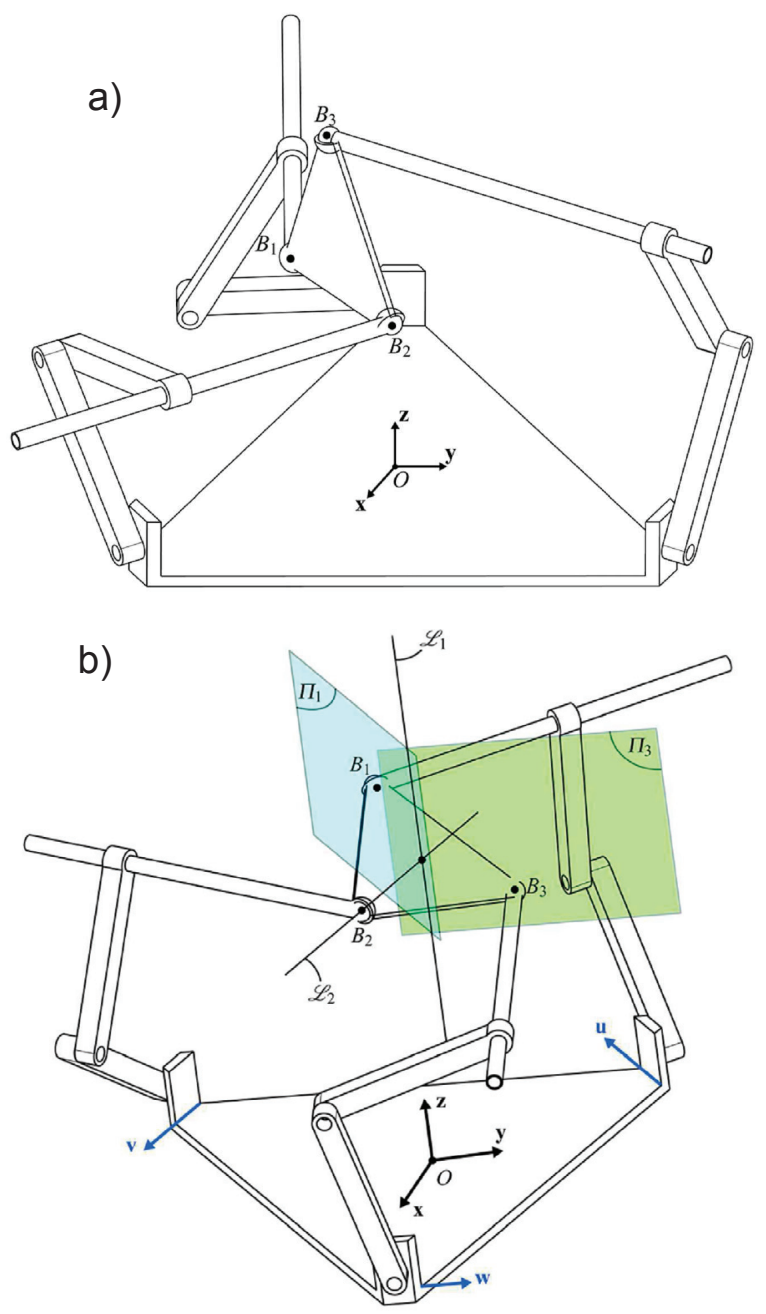

Fig. 3. a) A singular configuration of MEPaM where its moving platform is vertical; b) A singular configuration of MEPaM where lines $\mathcal{L}_{1}$ and $\mathcal{L}_{2}$ intersect

An interesting feature of the manipulator is that the parallel singularity is not dependent on the end-effector orientation. Moreover, the design of the device prevents the end-effector from reaching the parallel singularities thanks to the physical limits of the universal joints.

\section{Force controller for a haptic device}

The force controller [12] was applied to the MEPaM, as shown in Fig. 4. The symbols in the figure are: virtual environment impedance $\mathbf{Z}_{\text {env }}$, penetration of the avatar through the virtual environment $\mathbf{X}_{e n v}$, desired force $\mathbf{F}_{d}$, admittance $\mathbf{Z}_{h}^{-1}$, inverse Jacobian $\mathbf{J}^{T}$ (depending on the robot pose) which maps $\mathbf{F}_{d}$ into desired torque commands $\boldsymbol{\tau}_{d}$, which are applied to the haptic device. The torques to resist a given force $\mathbf{F}$ are dependent on the robot configuration.

Such type of controller requires the estimation of the endeffector pose $\mathbf{X}$. Usually, it is estimated using the information of the joints (such as in Fig. 4), but, in order to overpass the 


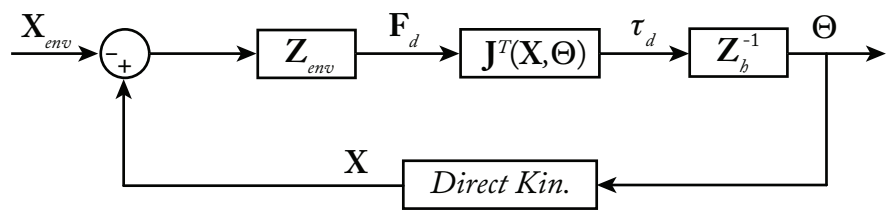

Fig. 4. The open-loop force control.

model errors and improve the accuracy, it is better to use vision and to measure the position of some robot elements as close as possible to the end-effector. The most efficient method would be to observe directly the end-effector. However, for an haptic device, the user hand may prevent this observation. To avoid this problem, we propose to observe the cylindrical links which are directly connected to the end-effector. In the next section, we disclose about the end-effector pose estimation based on leg observation.

\section{END-EFFECTOR POSE ESTIMATION USING LEG OBSERVATION}

\section{A. Line modeling}

A line $\mathcal{L}$ in $3 \mathrm{D}$ may be parameterized by its Binormalized Plücker coordinates [13]:

$$
\mathcal{L} \equiv\left({ }^{c} \underline{\mathbf{u}},{ }^{c} \underline{\mathbf{n}},{ }^{c} n\right)
$$

where ${ }^{c} \underline{\mathbf{u}}$ is a unit vector parameterizing the direction of the line $^{3},{ }^{c} \underline{\mathbf{n}}$ is a unit vector orthogonal to the line interpretation plane $\mathcal{L}$ (defined as the plane lying on the line $\mathcal{L}$ and passing through the camera frame origin) and ${ }^{c} n$ is a scalar superior or equal to zero. ${ }^{c} n$ and ${ }^{c} \mathbf{n}$ are linked through the relation ${ }^{c} n^{c} \underline{\mathbf{n}}={ }^{c} \mathbf{P} \times{ }^{c} \underline{\mathbf{u}}$ where ${ }^{c} \mathbf{P}$ is the location of a point $P$ on the line with respect to the camera frame.

The characteristic equation of such a line projected in the image plane is given in the camera frame by [13]:

$$
{ }^{c} \underline{\mathbf{n}}^{T c} \mathbf{P}=0
$$

With the matrix $\mathbf{K}$ formed by intrinsic parameters of the camera, one can obtain the line equation in pixel coordinates ${ }^{p} \underline{\mathbf{n}}$ from: In pixel coordinates, the line equation is given by:

$$
{ }^{p} \underline{\mathbf{n}}^{T p} \mathbf{p}=0
$$

Indeed, replacing ${ }^{p} \mathbf{p}$ with $\mathbf{K}^{c} \mathbf{p}$ in this expression yields:

$$
{ }^{p} \underline{\mathbf{n}}^{T} \mathbf{K}^{c} \mathbf{p}=0
$$

By comparing (3) and (5), one obtains

$$
{ }^{p} \underline{\mathbf{n}}=\frac{\mathbf{K}^{-T c} \underline{\mathbf{n}}}{\left\|\mathbf{K}^{-T c} \underline{\mathbf{n}}\right\|}, \underline{c} \underline{\mathbf{n}}=\frac{\mathbf{K}^{T p} \underline{\mathbf{n}}}{\left\|\mathbf{K}^{T p} \underline{\mathbf{n}}\right\|}
$$

${ }^{3}$ In the following of the paper, the superscript before the vector denotes the frame in which the vector is expressed (" $b$ " for the base frame, " $c$ " for the camera frame and " $p$ " for the prixel frame). If there is no superscript, the vector can be written in any frame.

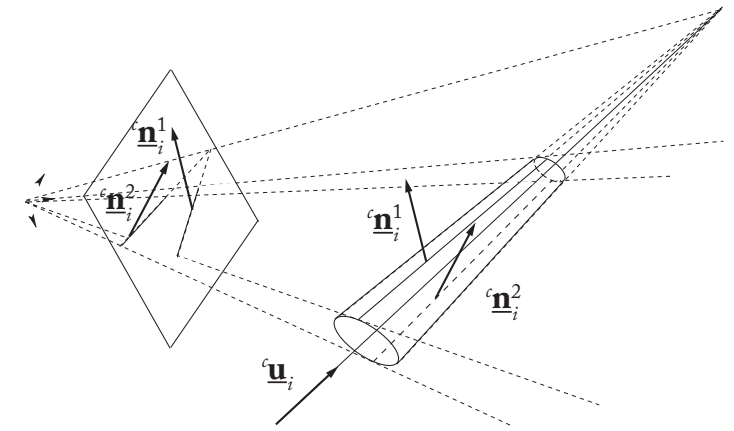

Fig. 5. A cylinder projected in the image

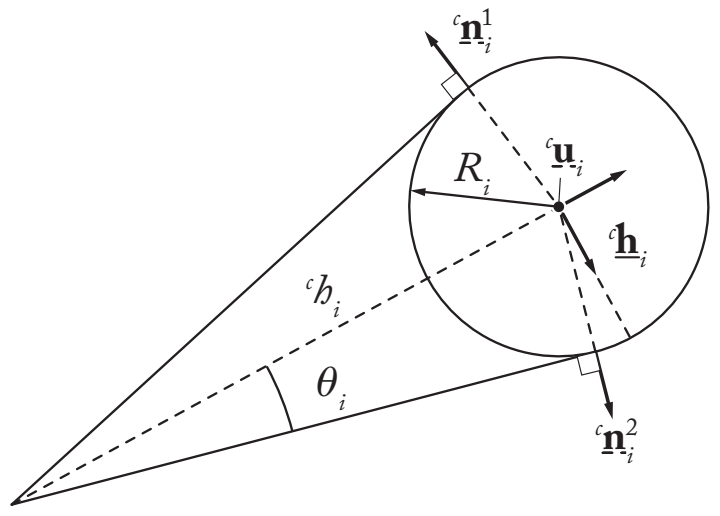

Fig. 6. Edges of a cylinder observed by vision

\section{B. Cylindrical leg observation}

The legs of parallel robots have usually cylindrical crosssections [1]. The edges of cylindrical leg $i$ are given, in the camera frame, by [14] (Figs. 5 and 6):

$$
\begin{aligned}
& { }^{c} \underline{\mathbf{n}}_{i}^{1}=-\cos \theta_{i}{ }^{c} \underline{\mathbf{h}}_{i}-\sin \theta_{i}{ }^{c} \underline{\mathbf{u}}_{i} \times{ }^{c} \underline{\mathbf{h}}_{i} \\
& { }^{c} \underline{\mathbf{n}}_{i}^{2}=+\cos \theta_{i}{ }^{c} \underline{\mathbf{h}}_{i}-\sin \theta_{i}{ }^{c} \underline{\mathbf{u}}_{i} \times{ }^{c} \underline{\mathbf{h}}_{i}
\end{aligned}
$$

where $\cos \theta_{i}=\sqrt{{ }^{c} h_{i}^{2}-R_{i}^{2}} /{ }^{c} h_{i}, \sin \theta_{i}=R_{i} /{ }^{c} h_{i}$ and $\left({ }^{c} \underline{\mathbf{u}}_{i},{ }^{c} \underline{\mathbf{h}}_{i},{ }^{c} h_{i}\right)$ are the Binormalized Plücker coordinates of the cylinder axis and $R_{i}$ is the cylinder radius.

From [14], the leg orientation can be defined in the camera frame by

$$
{ }^{c} \underline{\mathbf{u}}_{i}=\frac{{ }^{c} \underline{\mathbf{n}}_{i}^{1} \times{ }^{c} \underline{\mathbf{n}}_{i}^{2}}{\left\|\underline{\mathbf{n}}_{i}^{1} \times{ }^{c} \underline{\mathbf{n}}_{i}^{2}\right\|}
$$

It is known that each cylinder edge is a spatial line parameterized by the Plücker coordinates $\left({ }^{c} \underline{\mathbf{u}}_{i},{ }^{c} \underline{\mathbf{n}}_{i}^{j},{ }^{c} n_{i}^{j}\right)$ (Fig 5). Moreover, any point $A_{i}$ (of coordinates ${ }^{c} \mathbf{A}_{i}$ in the camera frame) located on the axis of the cylinder is at the distance $R_{i}$ from the edge of the cylinder.

Consequently, any edge of a cylinder edge is totally defined by the following relations, valid in any frame:

$$
\begin{gathered}
{ }^{c} \underline{\mathbf{n}}_{i}^{j T c} \mathbf{A}_{i}=-R_{i} \\
{ }^{c} \underline{\mathbf{n}}_{i}^{j T c} \underline{\mathbf{n}}_{i}^{j}=1 \\
{ }^{c} \underline{\mathbf{u}}_{i}^{T c} \underline{\mathbf{n}}_{i}^{j}=0
\end{gathered}
$$




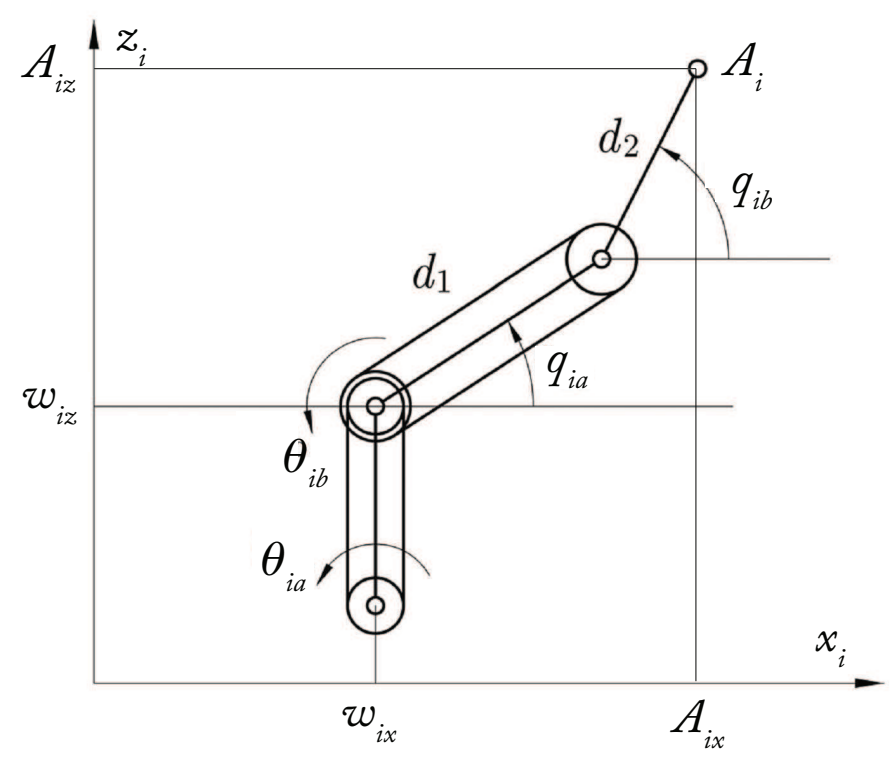

Fig. 7. The belt-pulley transmission of MEPaM for the leg $i$

The vector ${ }^{c} \mathbf{h}_{i}={ }^{c} h_{i}{ }^{c} \underline{\mathbf{h}}_{i}$ can be computed using the edges of the cylindrical leg $i$ too, and it is given by

$$
{ }^{c} \mathbf{h}_{i}={ }^{c} \mathbf{D}_{i} \times{ }^{c} \underline{\mathbf{u}}_{i}
$$

where ${ }^{c} \mathbf{D}_{i}$ is the position of the point $D_{i}$ in the camera frame, which is the closest point of the axis of the $i$-th leg to the camera. It is given by

$$
{ }^{c} \mathbf{D}_{i}=\frac{R_{i}}{\sin \left(\theta_{i}\right)} \cdot \frac{{ }^{c} \mathbf{n}_{i}^{1}+{ }^{c} \mathbf{n}_{i}^{2}}{\left\|{ }^{c} \mathbf{n}_{i}^{1}+{ }^{c} \mathbf{n}_{i}^{2}\right\|}
$$

\section{MEPaM kinematics based on Plücker coordinates}

The objective of the work is to use the information of the leg Plücker coordinates instead of the joint angles to estimate the MEPaM end-effector pose, therefore the forward kinematic problem has to be solved using them.

The Figs. 7 and 8 are useful to introduce the kinematics of MEPaM. Figure 7 shows the kinematic representation of the MEPaM legs and Fig. 8 shows the frame assignment for the driving planes: three fixed frames, $\mathcal{F}_{1}\left(O_{1}, \vec{X}_{1}, \vec{Y}_{1}, \vec{Z}_{1}\right), \mathcal{F}_{2}$ $\left(\mathrm{O}_{2}, \vec{X}_{2}, \vec{Y}_{2}, \vec{Z}_{2}\right)$ and $\mathcal{F}_{3}\left(O_{3}, \vec{X}_{3}, \vec{Y}_{3}, \vec{Z}_{3}\right)$, are attached to the base in an equilateral triangle formation, while a moving frame $\mathcal{F}_{4}\left(B_{1}, \vec{X}_{4}, \vec{Y}_{4}, \vec{Z}_{4}\right)$ is attached to the triangular end-effector of side length $d_{3}$. $\mathbf{A}_{i}$ and $\mathbf{B}_{i}$ are the respective Cartesian coordinate vectors of the endpoints of the $i$-th leg $A_{i}$ and $B_{i}$. The upper-left index indicates the frame in which the vector is expressed.

Since each cylindrical leg attached to the triangular platform is orthogonal to the plane of the corresponding leg, we have

$$
{ }^{1} \mathbf{B}_{1}=\left[\begin{array}{c}
{ }^{1} A_{1 x} \\
l_{1} \\
{ }^{1} A_{1 z}
\end{array}\right],{ }^{2} \mathbf{B}_{2}=\left[\begin{array}{c}
{ }^{2} A_{2 x} \\
l_{2} \\
{ }^{2} A_{2 z}
\end{array}\right],{ }^{3} \mathbf{B}_{3}=\left[\begin{array}{c}
{ }^{3} A_{3 x} \\
l_{3} \\
{ }^{3} A_{3 z}
\end{array}\right]
$$

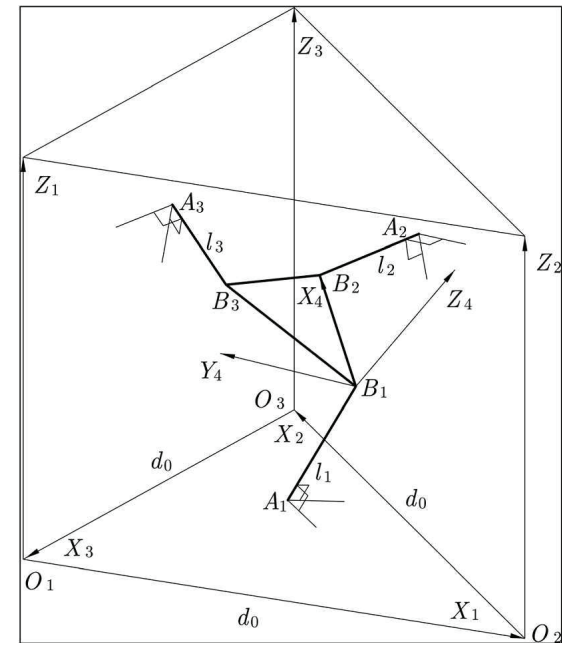

Fig. 8. The end-effector of MEPaM connected to $A_{1}, A_{2}$ and $A_{3}$

where $l_{i}$ is the length of the $i$-th cylindrical leg and ${ }^{i} A_{i x}$ and ${ }^{i} A_{i z}$ are the $x$ and $z$ coordinates of the point $A_{i}$ in the local frame $\mathcal{F}_{i}$ (Fig. 7).

In order to estimate MEPaM end-effector pose, we first need to estimate through vision the position of the points $A_{i}$ (Figs. 7 and 8) which are necessary in the relation (15). We have

$$
\left[\begin{array}{c}
{ }^{i} \mathbf{A}_{i} \\
1
\end{array}\right]={ }_{0}^{i} \mathbf{T} \quad\left[\begin{array}{c}
{ }^{0} \mathbf{A}_{i} \\
1
\end{array}\right]
$$

The values of ${ }_{0}^{i} \mathbf{T}$ are given by the robot design, and are equal to:

$$
{ }_{0}^{1} T=\left[\begin{array}{cccc}
0 & 1 & 0 & 0 \\
-1 & 0 & 0 & r_{1} \\
0 & 0 & 1 & 0 \\
0 & 0 & 0 & 1
\end{array}\right]
$$

$$
{ }_{0}^{2} T=\left[\begin{array}{cccc}
\mathrm{C}\left(\frac{7}{6} \pi\right) & \mathrm{S}\left(\frac{7}{6} \pi\right) & 0 & \frac{r_{2}}{2} \mathrm{C}\left(\frac{7}{6} \pi\right)-\sqrt{3} \frac{r_{2}}{2} \mathrm{~S}\left(\frac{7}{6} \pi\right) \\
-\mathrm{S}\left(\frac{7}{6} \pi\right) & \mathrm{C}\left(\frac{7}{6} \pi\right) & 0 & -\frac{r_{2}}{2} \mathrm{~S}\left(\frac{7}{6} \pi\right)-\sqrt{3} \frac{r_{2}}{2} \mathrm{C}\left(\frac{7}{6} \pi\right) \\
0 & 0 & 1 & 0 \\
0 & 0 & 0 & 1
\end{array}\right]
$$

${ }_{0}^{3} T=\left[\begin{array}{cccc}\mathrm{C}\left(\frac{11}{6} \pi\right) & \mathrm{S}\left(\frac{11}{6} \pi\right) & 0 & \frac{r_{3}}{2} \mathrm{C}\left(\frac{11}{6} \pi\right)+\sqrt{3} \frac{r_{3}}{2} \mathrm{~S}\left(\frac{11}{6} \pi\right) \\ -\mathrm{S}\left(\frac{11}{6} \pi\right) & \mathrm{C}\left(\frac{11}{6} \pi\right) & 0 & -\frac{r_{3}}{2} \mathrm{~S}\left(\frac{11}{6} \pi\right)+\sqrt{3} \frac{r_{3}}{2} \mathrm{C}\left(\frac{11}{6} \pi\right) \\ 0 & 0 & 1 & 0 \\ 0 & 0 & 0 & 1\end{array}\right]$

where $r_{1}, r_{2}$ and $r_{3}$ are the distances between the base frame with respect to the origin of the frames $\mathcal{F}_{1}, \mathcal{F}_{2}$ and $\mathcal{F}_{3}$ respectively, while ${ }^{0} \mathbf{A}_{i}$ will be estimated through vision as the intersection between the line passing through $i$-th leg and its corresponding perpendicular plane shown in Fig. 8. Note that in these equations, $\mathrm{S}(q)$ stands for $\sin q$ and $\mathrm{C}(q)$ stands for $\cos q$.

To compute the positions of points $A_{i}$, we use the following equations. Given a line expressed in Plücker coordinates as $[\underline{\mathbf{u}}, \underline{\mathbf{h}}, h]$ and a plane expressed in Plücker coordinates too 
as $[N \mid n]^{4}$, the general formula which gives the point of intersection between them is [15]

$$
\hat{\mathbf{A}}_{i}=(-(h \underline{\mathbf{h}}) \times N-n \underline{\mathbf{u}} \mid \underline{\mathbf{u}} N)
$$

where $\hat{\mathbf{A}}_{i}$ are the Plücker coordinates of the point $A_{i}$ (equivalent to the homogeneous coordinates of the point $A_{i}$ ).

From the robot design parameters, the transformation matrices between frames $\mathcal{F}_{1}, \mathcal{F}_{2}$ and $\mathcal{F}_{3}$ are given by

$$
{ }_{2}^{1} \mathbf{T}={ }_{3}^{2} \mathbf{T}={ }_{1}^{3} \mathbf{T}=\left[\begin{array}{cccc}
\cos (2 \pi / 3) & -\sin (2 \pi / 3) & 0 & d_{0} \\
\sin (2 \pi / 3) & \cos (2 \pi / 3) & 0 & 0 \\
0 & 0 & 1 & 0 \\
0 & 0 & 0 & 1
\end{array}\right]
$$

The position of all the vertices of the platform can be transformed into $\mathcal{F}_{1}$,

$$
{ }^{1} \mathbf{B}_{i}={ }_{i}^{1} \mathbf{T}^{i} \mathbf{B}_{i}
$$

for $i=2,3$. The geometric constraints on the device are given by

$$
\left\|{ }^{1} \mathbf{B}_{i}-{ }^{1} \mathbf{B}_{j}\right\|=d_{3}^{2}
$$

for $i=1,2,3$ and $j=i+1(\bmod 3)$, where $d_{3}$ is the (constant) side length of the platform.

The constraint equations, Eqs. (23), contain only three variables $l_{i}, i=1,2,3$, and can be written in the form

$$
\begin{aligned}
& D_{2} l_{2}^{2}+D_{1} l_{2}+D_{0}=0 \\
& E_{2} l_{3}^{2}+E_{1} l_{3}+E_{0}=0 \\
& F_{2} l_{3}^{2}+F_{1} l_{3}+F_{0}=0
\end{aligned}
$$

where $D_{j}, E_{j}, F_{j}(j=0,1,2)$, which are not developed here for brevity, are functions of $l_{1}, l_{2}$ and $l_{3}$, respectively, as well as $d_{3}, A_{i x}$ and $A_{i z}(i=1,2,3)$. By means of dialytic elimination, the system of Eqs. (24), (25), (26) can be reduced to a univariate polynomial of order four in the variable $l_{1}$

$$
G_{4} l_{1}^{4}+G_{3} l_{1}^{4}+G_{2} l_{1}^{2}+G_{1} l_{1}^{2}+G_{0}=0
$$

Once Eq. (27) has been solved for $l_{1}$, substitution of the result into Eqs. (24), (25), (26) will allow for determination of $l_{2}$ and $l_{3}$. Solutions of $l_{i}$ for $i=1,2,3$ are used to estimate the end-effector pose. The position of the end-effector is simply the origin of $\mathcal{F}_{4}$, given by ${ }^{1} \mathbf{B}_{1}=\left[A_{1 x}, l_{1}, A_{1 z}\right]^{T}$. The endeffector orientation is parameterized by ${ }_{4}^{1} \mathbf{Q}=[\mathbf{i}, \mathbf{j}, \mathbf{k}]$ where

$$
\begin{gathered}
\mathbf{i}=\frac{{ }^{1} \mathbf{B}_{2}-{ }^{1} \mathbf{B}_{1}}{\left\|{ }^{1} \mathbf{B}_{2}-{ }^{1} \mathbf{B}_{1}\right\|} \\
\mathbf{j}=\frac{\left({ }^{1} \mathbf{B}_{3}-{ }^{1} \mathbf{B}_{1}\right)-\mathbf{i i}^{T}\left({ }^{1} \mathbf{B}_{3}-{ }^{1} \mathbf{B}_{1}\right)}{\left\|\left({ }^{1} \mathbf{B}_{3}-{ }^{1} \mathbf{B}_{1}\right)-\mathbf{i i}^{T}\left({ }^{1} \mathbf{B}_{3}-{ }^{1} \mathbf{B}_{1}\right)\right\|} \\
\mathbf{k}=\mathbf{i} \times \mathbf{j}
\end{gathered}
$$

\footnotetext{
${ }^{4}$ Given the Cartesian equation of a plane $a x+b y+c z+d=0$, its Plücker coordinates are $[N \mid n]$ where $N=(a, b, c)$ and $n=d$
}

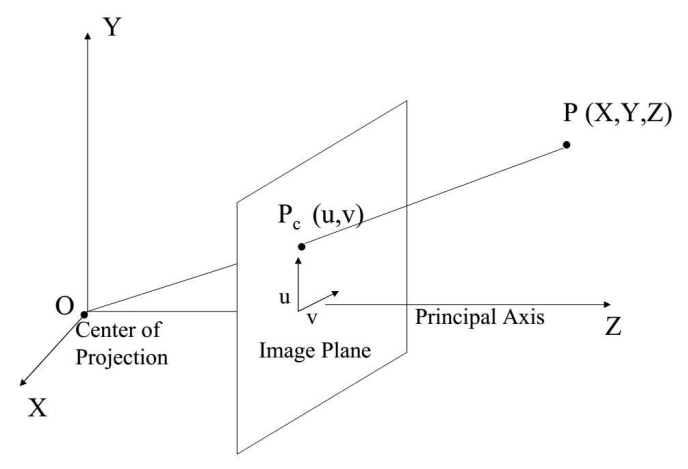

Fig. 9. A pinhole camera model

\section{Singularities of the new model}

It can be shown that, from [10], the standard forward kinematic problem (based on the encoder values $\Theta$ ) have the same inputs as the forward kinematic problem based on the leg pose visual estimation shown in subsection III-C, which are the positions of points $A_{i}$. Even if the positions of points $A_{i}$ are estimated through different manners (through the encoder positions and the leg geometric parameters in [10] while it is through vision in the current work), the structure of the forward kinematic model remains the same. Therefore, the singularities of the new model based on the Plücker coordinates are the same of the ones shown in [10] (the parallel singularities disclosed in Section II-B).

\section{Simulation Results}

In this section, some examples of simulation results are presented to show the efficiency of the end-effector pose through the leg observation. These results have been got with a model in Adams of MEPaM with the following nominal parameters (in meters):

$r_{i}=0.165$;

$d_{1}=[0.1375,0.1375,0.1375]$;

$d_{2}=[0.1375,0.1375,0.1375]$

$w_{x}=0, w_{z}=0.110$.

Moreover, the radius of the observed cylinder is equal to $1 \mathrm{~cm}$. We simulated a motion of MEPaM. The initial position and orientation of the platform is: $\mathbf{X}_{d 0}=[0,0,0.180,-$ pi $/ 4,0,0]$. Here, the orientation of the end-effector is described by using the $Z Y X$ Euler angles convention (roll-pitch-yaw angles $\phi$, $\theta$ and $\psi$ ). The trajectories given to the actuators (whose angles are shown in Fig. 7) as input are: $\dot{q}_{1 a}=0.001 \sin (t)$, $\dot{q}_{1 b}=0.001 \sin (t), \dot{q}_{2 a}=0.001 \sin (t), \dot{q}_{2 b}=0.1 \sin (t)$, $\dot{q}_{3 a}=0.05 \sin (t), \dot{q}_{3 b}=0.01 \sin (t)$.

We simulated the camera located at the centre of the base frame $\mathcal{F}_{0}$ (with the camera plane parallel to the $x_{0} O y_{0}$ plane) by using a pinhole camera model because it is simple to implement and is a good approximation of real cameras. In the Fig. 9, a camera with $O$ as center of projection and the principal axis parallel to $Z$ axis is shown. The distance between $O$ and the image plane is the focal length $f$. The 3D point $P=(X, Y, Z)$ is projected on the image plane at coordinates $P_{c}=(u, v)$. The parameters of the camera used for the simulations are: 


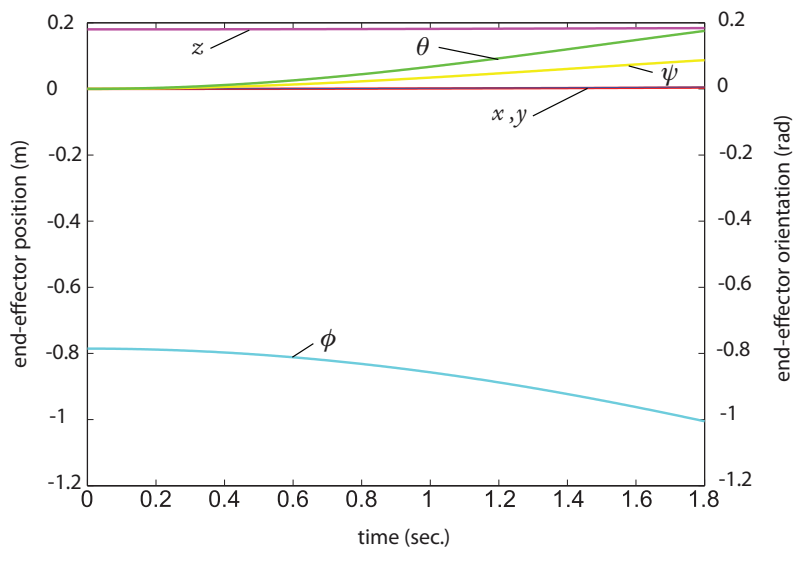

Fig. 10. The end-effector position and orientation vector computed with the new pose estimation method without any noise.

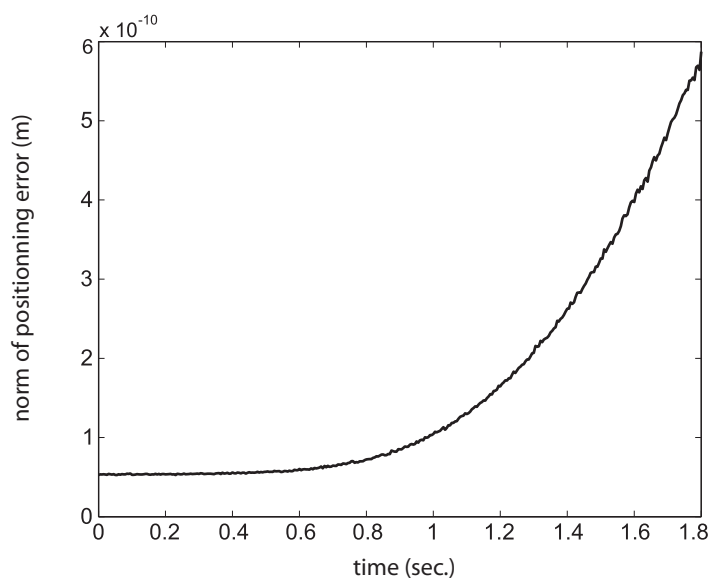

Fig. 11. The error norm between the computed and real end-effector positions without any noise.

- focal length with respect to $u=10^{3}$ pixels;

- focal length with respect to $v=10^{3}$ pixels;

- principle point in image along $u=1024$ pixels;

- principle point in image along $v=768$ pixels.

Without adding any noise, we obtained the results shown in Figs. 10, 11 and 12: the norm of the error between the computed position of the end-effector and the real one is very low (around $10^{-10}$ meters) as well as the norm of the error of the orientation (around $10^{-7}$ radians), therefore the new method for the pose estimation computes the pose in a very good way.

Then, we added a measurement noise that is introduced like thereafter. The extraction of the Plücker coordinates of the leg line is based on the equations of the leg edges. In the simulation, they are projected to the image plane and converted from meter to pixel. Then, the edge line intersections with image boundary are computed: the coordinates of the intersection points have to be rounded due to the pixel accuracy. A new equation of the edge line is then recomputed taking into account the error introduced in the intersection points between

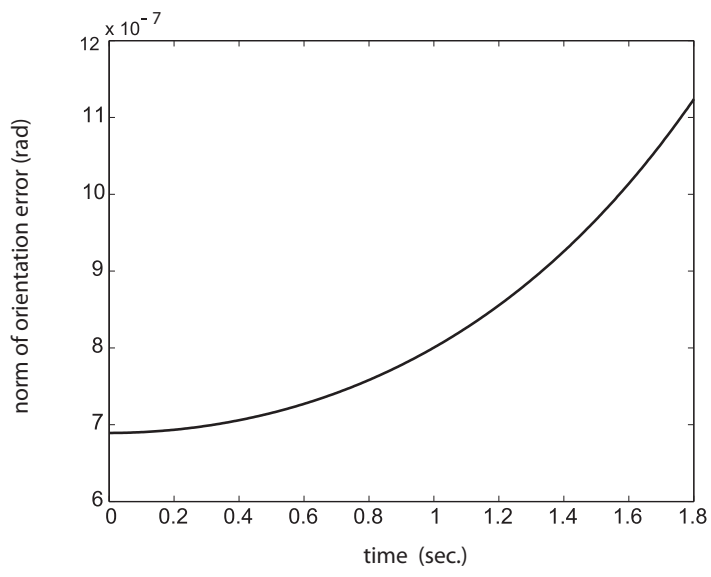

Fig. 12. The error norm between the computed and real end-effector orientations without any noise.

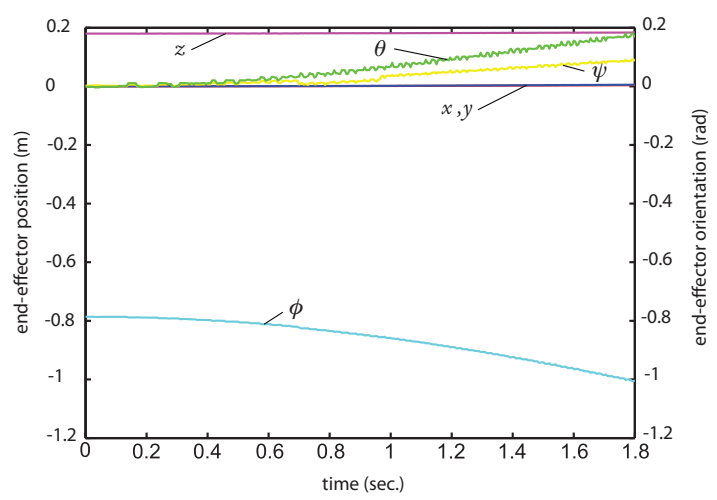

Fig. 13. The end-effector position and orientation vector computed with the new pose estimation method with measurement noise.

the edge line and the image boundary. The measurements error chosen is given by a pixel accuracy equal to 1 . The results obtained adding noise are shown in Figs. 13, 14 and 15: it is possible to notice that the norm of the error of the position is in the order of $10^{-4} \mathrm{~m}$ and the norm of the error of the rotation is in the order of $10^{-2} \mathrm{rad}$, therefore the new method for the pose estimation shows a good robustness to measurement noise.

\section{COnclusions}

In this paper a new approach for end-effector pose estimation in the case of a parallel robot has been proposed. This method is based on the extraction of the Plücker coordinates of the leg center line and, unlike the old method based on the leg direction proposed in the previous papers, it is suitable to all the Parallel Kinematic Machines families, including parallel robots whose legs directions are constant even if the endeffector pose changes, like MEPaM. This new method has been applied to MEPaM and some simulation results (without any noise and with measurement noise as well) of the new pose estimator have shown its efficiency.

\section{REFERENCES}

[1] J.P. Merlet. Parallel Robots. Springer, 2nd edition, 2006. 


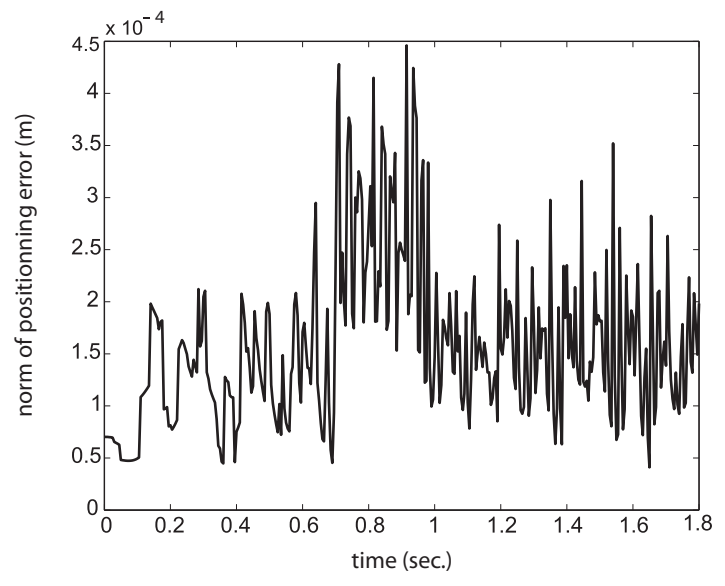

Fig. 14. The norm of the error between the computed end-effector position and the real one with measurement noise.

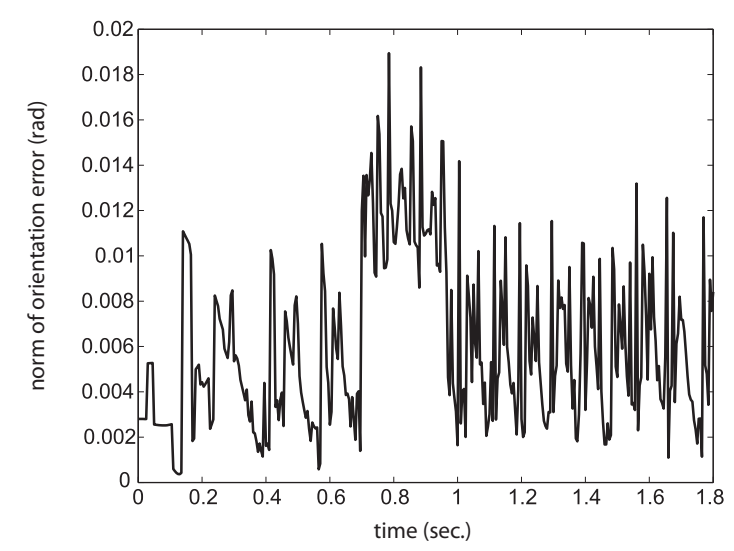

Fig. 15. The norm of the error between the computed end-effector rotation and the real one with measurement noise.

[2] B. Espiau, F. Chaumette, and P. Rives. A new approach to visual servoing in robotics. IEEE Transactions on Robotics and Automation, 8(3), 1992.

[3] R. Horaud, F. Dornaika, and B. Espiau. Visually guided object grasping. IEEE Transactions on Robotics and Automation, 14(4):525-532, 1998.

[4] P. Martinet, J. Gallice, and D. Khadraoui. Vision based control law using 3D visual features. In Proceedings of the World Automation Congress, WAC96, Robotics and Manufacturing Systems, volume 3, pages 497502, Montpellier, France, May 1996.

[5] E. Ozgur, N. Andreff, R. Dahmouche, and P. Martinet. High speed parallel kinematic manipulator state estimation from legs observation. In Proceedings of the IEEE/RSJ International Conference on Intelligent Robots and Systems (IROS 2013), Tokyo Big Sight, Japan, 2013.

[6] N. Andreff, A. Marchadier, and P. Martinet. Vision-based control of a Gough-Stewart parallel mechanism using legs observation. In Proceedings of the IEEE International Conference on Robotics and Automation, ICRA'05, pages 2546-2551, Barcelona, Spain, April 18222005 .

[7] V.E. Gough and S.G. Whitehall. Universal tyre test machine. In Proceedings of the FISITA 9th International Technical Congress, pages 117-317, May 1962.

[8] E. Ozgur, N. Andreff, and P. Martinet. Dynamic control of the quattro robot by the leg edgels. In Proceedings of the IEEE International Conference on Robotics and Automation, ICRA11, Shanghai, China, May 9-13 2011.

[9] N. Andreff and P. Martinet. Vision-based kinematic modelling of some parallel manipulators for control purposes. In Proceedings of EuCoMeS, the First European Conference on Mechanism Science, Obergurgl, Austria, 2006.

[10] C. Chen, T. Gayral, S. Caro, D. Chablat, G. Moroz, and S. Abeywardena. A Six Degree of Freedom Epicyclic-Parallel Manipulator. 2012.

[11] S. Caro, G. Moroz, T. Gayral, D. Chablat, and C. Chen. Singularity Analysis of a Six-DOF Parallel Manipulator Using Grassmann-Cayley Algebra and Grobner Bases. Symposium on Brain, Body and Machine, Montreal, QC.,Canada, 2010.

[12] L. Villani and J. De Schutter. Handbook of Robotics, chapter Ch7: Force Control, pages 161-186. Springer, 2008.

[13] N. Andreff, B. Espiau, and R. Horaud. Visual servoing from lines. International Journal of Robotics Research, 21(8):679-700, 2002.

[14] N. Andreff, T. Dallej, and P. Martinet. Image-based visual servoing of gough-stewart parallel manipulators using legs observation. International Journal of Robotics Research, 26(7):677-687, 2007.

[15] J.M. Selig. Geometric fundamentals of robotics, 2005. Springer, 2nd edition. 